\title{
Phylogenetic Analysis of the Three Color Variations of the Sea Cucumber Apostichopus japonicus
}

\author{
Jihoon Jo1, Chunsik Park², Munhwan Kim³ and Chungoo Park ${ }^{1 *}$
}

${ }^{1}$ School of Biological Sciences and Technology, Chonnam National University, Gwangju, 500-757, Republic of Korea

${ }^{2}$ College of Veterinary Medicine, Chonnam National University, Gwangju, 500-757, Republic of Korea

${ }^{3}$ Academic and Student Affairs Division, Chonnam National University, Yeosu, 550-749, Republic of Korea

\begin{abstract}
The economic value of Apostichopus japonicus products is primarily determined by their dorsal/ventral color variation (red, green, or black), yet the taxonomic relationships between these color variants are not clearly understood. By performing numerous phylogenetic analyses of the Stichopodidae family, based on nucleotide sequence comparisons of the cytochrome $c$ oxidase subunit 1 (COI) and 16S rRNA gene sequences, we observed that these three color variants exhibit very low levels of sequence divergence and are not monophyletic. In this paper, we propose that the different dorsal/ventral color types of $A$. japonicus belong to a single species.
\end{abstract}

Keywords: Sea cucumber; Apostichopus japonicas; Body-color variations; Phylogenetic analysis

\section{Introduction}

The sea cucumber is a commercially and medically important member of the phylar group Echinodermata, which is one of the most abundant and ecologically successful marine-invertebrate clades. More than 1,250 species of sea cucumber have been identified on the sea floor worldwide and approximately 20 of them are edible. One especially important species is the sea cucumber (Apostichopus japonicas, Selenka 1867), which is mostly found off the coasts of Northeast Asia including Northern China, Korea, Japan, and Far East Russia, as it is used as a source of seafood and in traditional medicine[1,2]. Sea cucumbers also can regenerate their missing organs within a few months, and this has generated particular interest among the biomedical-research field. One of the most important traits of the Apostichopus japonicus species is body color. According to their dorsal/ventral color types, three variants (red, green, and black) have been found, and they have distinct morphological differences (e.g., ossicle shape, habitat preference, spawning period, and polian vesicles) [1,3].

The body color of A. japonicus affects the price and taste of the corresponding products, whereby the rare red-color type is the most favored and expensive. The recent global exploitation of sea cucumbers to meet consumer demand, however, continues to motivate a rising conservation concern $[4,5]$. To establish genetic and breeding systems for sea-cucumber aquaculture as part of the overall management of natural resources, it is clearly necessary to clarify the taxonomic relationships among these color variants; to this end, several studies have sought to address this issue. For example, using the mitochondrial genomes of A. japonicus, observed very low levels of divergence among the three color variants, indicating that they belong to a single species; furthermore, they also showed that there are no obvious phylogenetic relationships among the geographically adjacent individuals, thereby suggesting the non-monophyletic origin of the three color variants of A. japonicas [6,7]. However, there are a number of caveats that should be considered when interpreting and generalizing these results. First, simple comparisons of the mean genetic distances between interspecific and intraspecific divergences are not adequate for the assessment of phylogenetic relationships and estimated the mean pair-wise sequence differences $(0.67 \%-0.74 \%)$ among the three color variants of $A$. japonicus based on the $p$-distances method [8], and then compared them to those (1.3\% intraspecific and $16.9 \%$ interspecific divergences) that are based on the Kimura 2-parameter method [9] among the species of the Stichopodidae family. Without the adjustment of the estimation methods and the completion of comprehensive phylogenetic analyses, the authors concluded that the three color variants of $A$. japonicus belong to a single species, which can lead to misleading or incorrect results. Second, the selection of outgroups for phylogenetic analyses is an important factor because the inappropriate selection of an outgroup can affect the ingroup topology [10,11]. Several studies have therefore proposed that a multiple-outgroup approach can solve this problem by improving the accuracy of a phylogenetic estimation; where a single outgroup is used, the tree topology like that of the three color variants of $A$. japonicas that was inferred by Zhang can be distorted $[12,13]$. In this study, to verify and better understand the taxonomic status of the three color variants of A. japonicus, we reconstructed the phylogenetic trees by using the sequences of partial cytochrome c oxidase subunit $1(C O I)$ and $16 S$ rRNA genes from A. japonicus samples including our new sequences, with 17 Stichopodidae species used as the outgroups [14]. The maximum-likelihood (ML), neighborjoining (NJ), and maximum-parsimony (MP) methods all support the same tree topology, whereby (1) red-, green-, and black-color variants are clustered in the same group; and (2) the color variants are not monophyletic. Our results confirm the taxonomic statuses of the three color variants of $A$. japonicus, and they will help to facilitate the rapid growth of the sea cucumber aquaculture industry and its breeding programs.

\section{Materials and Methods}

\section{Sample collection and DNA preparation}

We obtained all three of the body-color-types of wildlife specimens

*Corresponding author: Chungoo Park, School of Biological Sciences and Technology, Chonnam National University, Gwangju, Republic of Korea, Tel: +8262-530-1913; E-mail: chungoo@jnu.ac.kr

Received January 28, 2016; Accepted February 26, 2016; Published March 10 2016

Citation: Jo J, Park C, Kim M, Park C (2016) Phylogenetic Analysis of the Three Color Variations of the Sea Cucumber Apostichopus japonicus. J Aquac Res Development. 7: 418. doi:10.4172/2155-9546.1000418

Copyright: ( $2016 \mathrm{Jo} \mathrm{J}$, et al. This is an open-access article distributed under the terms of the Creative Commons Attribution License, which permits unrestricted use, distribution, and reproduction in any medium, provided the original author and source are credited. 
Citation: Jo J, Park C, Kim M, Park C (2016) Phylogenetic Analysis of the Three Color Variations of the Sea Cucumber Apostichopus japonicus. J Aquac Res Development. 7: 418. doi:10.4172/2155-9546.1000418

of the sea cucumber A. japonicus from the same geographic region near the coast of Geomun-do in South Korea $\left(34^{\circ} 1^{\prime} 35^{\prime \prime} \mathrm{N}, 127^{\circ} 18^{\prime} 45^{\prime \prime} \mathrm{E}\right)$. The total genomic DNA samples of the three color variants were extracted from the body wall. Approximately $5 \mathrm{~g}$ of the body wall tissue was dissected and digested for $1 \mathrm{~h}$ at $65^{\circ} \mathrm{C}$ in $10 \mathrm{ml}$ of CTAB (cetyl trimethylammonium bromide) lysis buffer ( $2 \%$ CTAB, $1.4 \mathrm{M} \mathrm{NaCl}$, $20 \mathrm{mM}$ EDTA, $100 \mathrm{mM}$ Tris- $\mathrm{HCl}$, and $\mathrm{pH} 8.0$ ) and $50 \mu \mathrm{l}$ of RNase A $(20 \mathrm{mg} / \mathrm{ml})$. The solution was extracted twice with phenol/chloroform. The DNA was precipitated with isopropanol and ammonium acetate and finally dissolved in TE buffer (10 mM Tris-HCl, $1 \mathrm{mM}$ EDTA, $\mathrm{pH}$ 8.0).

\section{PCR and sequencing}

To amplify two short fragments of the partial COI and $16 S$ rRNA from the mitochondrial genome, we used the following two pairs of universal primers: COIeF (5' - ATA ATG ATA GGA GGR TTT GG -3') and COIeR (5'- GCT CGT GTR TCT ACR TCC AT -3') [14], and 16 Sar (5'- CGC CTG TTT ATC AAA AAC AT -3') and 16Sbr (5'- CTC CGG TTT GAA CTC AGA TCA -3') [15]. The PCR reactions were performed with a SimpliAmp ${ }^{\mathrm{TM}}$ thermal cycler (Life Technologies). The first step was preceded by an initial denaturation for $5 \mathrm{~min}$ at $94^{\circ} \mathrm{C}$, and the following 40 cycles consisted of a $30 \mathrm{sec}$ denaturation at $94^{\circ} \mathrm{C}$, annealing for $30 \mathrm{sec}$ at $50^{\circ} \mathrm{C}\left(53^{\circ} \mathrm{C}\right.$ for the $16 \mathrm{~S}$ rRNA), and a 1 min extension at $72^{\circ} \mathrm{C}$. The last cycle was followed by a final extension for $10 \mathrm{~min}$ at $72^{\circ} \mathrm{C}$. The reaction volume amounted to $50 \mu \mathrm{l}$ reactions containing $25 \mu$ lof $2 \times$ Ampmaster $^{\mathrm{TM}} \mathrm{HS}$-Taq (GeneAll), $18.5 \mu \mathrm{l}$ of sterile deionized water, $2 \mu \mathrm{l}$ of each primer $(10 \mathrm{pmol})$, and $50 \mathrm{ng}$ of genomic DNA. All of the PCR products were purified using the QIAquick PCR purification kit (QIAGEN), and they were sequenced using the ABI BigDye Terminator v3.1 Cycle Sequencing Kits on an ABI 3730xl DNA Analyzer (Applied Biosystems). All of the newly acquired sequences were deposited into the GenBank (KT625443, KT625444, and KT625445 for COI; KT724356, KT724357, and KT724358 for $16 S$ rRNA).

\section{Phylogenetic analysis}

The COI and $16 S$ rRNA gene sequences from the 17 Stichopodidae species (Apostichopus japonicus, Astichopus multifidus, Australostichopus mollis, Isostichopus badionotus, Isostichopus fuscus, Parastichopus californicus, Parastichopus parvimensis, Stichopus chloronotus, Stichopus herrmanni, Stichopus horrens, Stichopus monotuberculatus, Stichopus naso, Stichopus ocellatus, Stichopus vastus, Thelenota ananas, Thelenota anax, and Thelenota rubralineata) were retrieved from the GenBank, with the accession numbers AB525437, AB525760, AB525761, AY852281, EU294194, FJ594963, FJ594967, FJ594968, FJ906623, FJ986223, GU557147, GU557148, EU848293, EU822453, EU856598, EU856599, EU856667, EU848276, JN207495, AF486424, AF486425, AY153494, DQ777096, U32198, U32199, EU848298, EU856618, EU856619, EU856614, EU856693, EU856544, EU848278 to 281, EU856545, EU856634, EU856554, EU848282, JQ657263, EU856557, EU856559, EU856543, EU856586, EU848279, EU848280, FJ001809, EU856607, EU220814, EU856608, EU856680, EU848275, EU856622, EU856709, EU848258, EU848259, EU848261, AY700771, EU848257, EU848243, EU848292, EU848260, and EU822452. The gene sequences were aligned using ClustalW [16] with default parameters. The phylogenetic trees were constructed using the NJ, MP, and ML methods with the Kimura-2-parameter [9] correction and 1,000 bootstrap replications. MEGA version 6 [17] was used for these evolutionary analyses
(A)

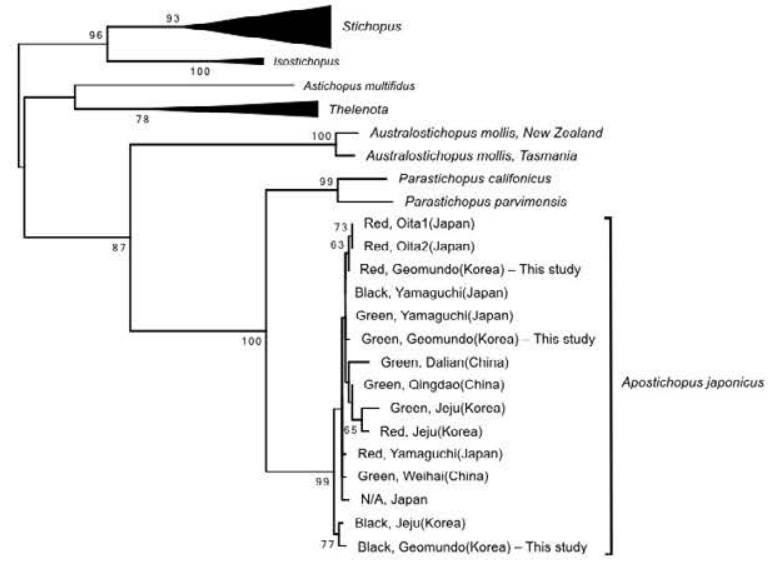

(B)

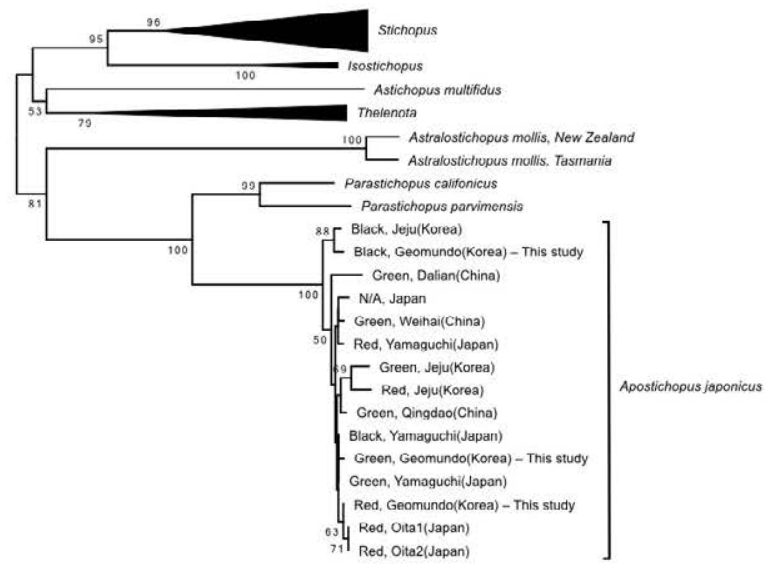

(C)

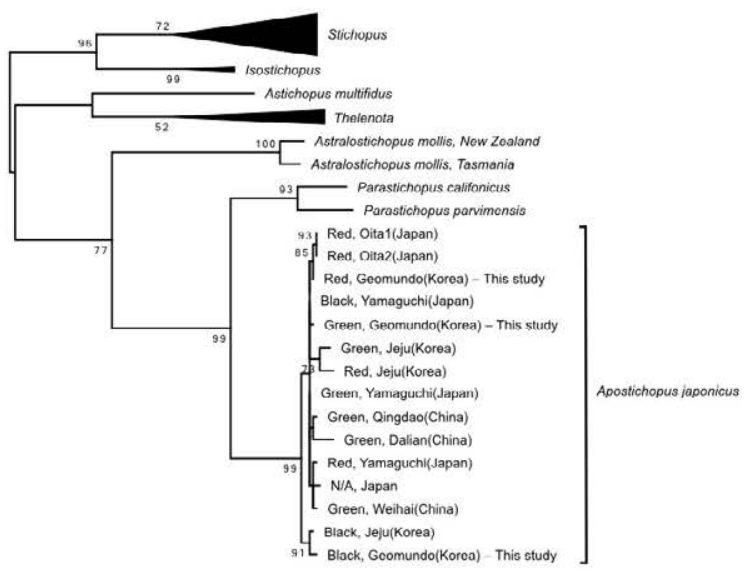

Figure 1: Phylogenetic trees of $\mathrm{CO}$ genes from the Stichopodidae family (A) The maximumlikelihood tree. Each branch length was measured by the number of substitutions per site. (B) The neighbor-joining tree. The evolutionary distance was computed using the Kimura 2- parameter method, and a unit of the number of base substitutions per site was used. (C) The maximum-parsimony tree. Bootstrap percentage in $\%$ of 1,000 replications was shown on the interior branches with support when it is higher than or equal to $50 \%$. Several clades within the same genus (depicted as black wedges) were collapsed. 
(A)

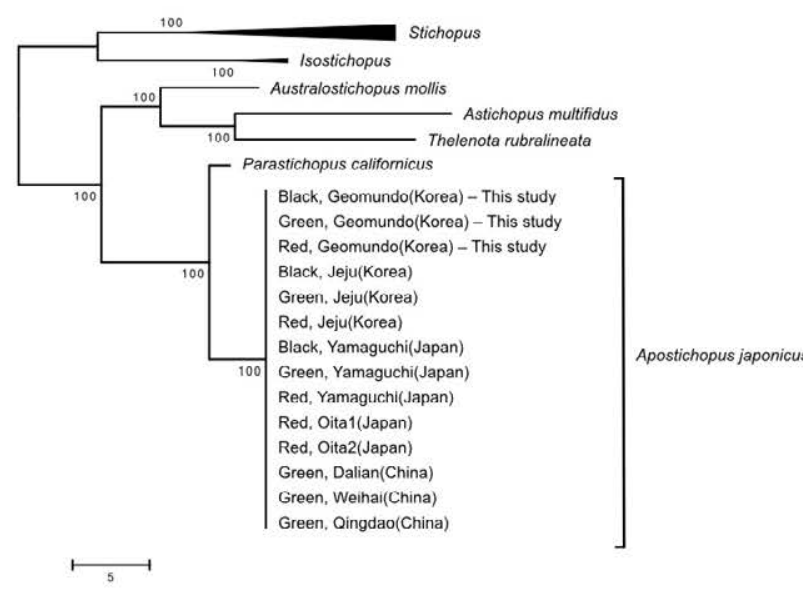

(B)

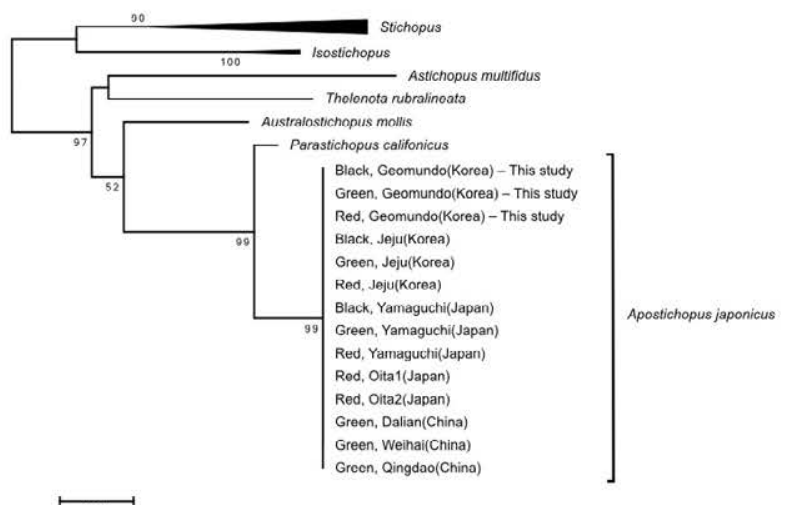

(C)

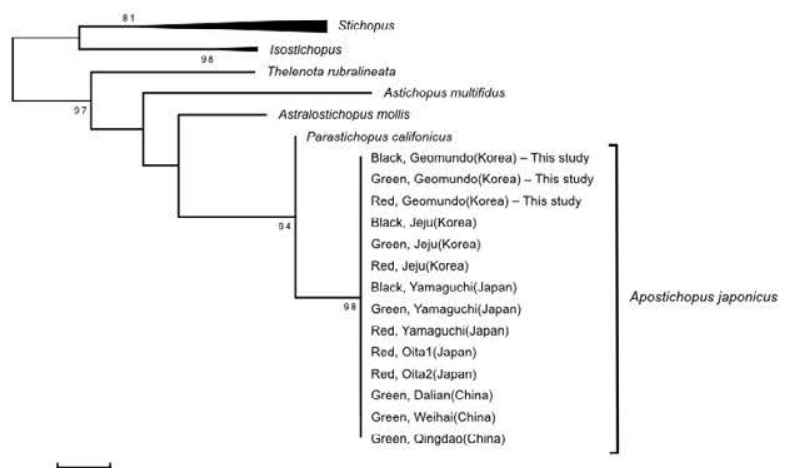

Figure 2: Phylogenetic trees of $16 \mathrm{~S}$ rRNA gene from the Stichopodidae family. (A) The maximum-likelihood tree. Each branch length was measured by the number of substitutions per site. (B) The neighbor-joining tree. The evolutionary distance was computed using the Kimura 2-parameter method, and a unit of the number of base substitutions per site was used. (C) The maximum-parsimony tree. Bootstrap percentage in $\%$ of 1,000 replications was shown on the interior branches with support when it is higher than or equal to $50 \%$. Several clades within the same genus (depicted as black wedges) were collapsed.

\section{Results and Discussion}

Because the COI barcode sequences have proved to be useful in the process of identifying and discovering species in Echinodermata [18], the available genetic information and newly generated COI sequences were analyzed to reconstruct the phylogenetic trees. Using the ML, NJ, and MP methods, we reconstructed the phylogenetic trees of the COI genes. All of the methods group the three color variants of A. japonicus into a single cluster (Figure 1), and the robustness of this cluster is strongly supported by the bootstrap values of $\geq 99 \%$ (Figure 1); Furthermore, the three color variants of A. japonicus have a very short branch length compared with other Stichopodidae groups. We also confirmed that Parastichopus is the closest genus (with bootstrap values of $\geq 99 \%$ ) to the Apostichopus species (Figure 1), which is consistent with a previous study [7]. The same pattern was observed when we examined the phylogenetic trees of the $16 \mathrm{~S}$ rRNA genes that contain species-specific signature sequences that are useful for identifying species (Figure 2) [19,20]. These results confirm and demonstrate convincingly that the three different dorsal/ventral color types of $A$. japonicus belong to a single species.

More than five decades ago, Choe and Oshima posited that the redcolor A. japonicus is morphologically, physiologically, and ecologically different from the green-color variant [1]. Our phylogenetic trees show that the A. japonicus population is divided into two clades with strong support (bootstrap values > 75\%) (Figure 1), as follows: one clade contains two (out of three) black individuals, while the other contains mixed individuals (one black, five red, and six green). Although three out of the five red individuals are clustered together with the bootstrap values of $>60 \%$, the remaining two red individuals are nested separately within the other distinct clades. The same pattern was observed for the green individuals (Figure 1); however, this evidence disappeared when the $16 \mathrm{~S}$ rRNA genes were used to construct the trees (Figure 2) because the $16 S$ rRNA sequences are too conserved to explore the intraspecies diversity of $A$. japonicus. Next, we investigated whether the hydrographic process and geographic distance shape the genetic variability and population structure of the sea cucumber A. japonicus. We collected a total of $15 \mathrm{~A}$. japonicus sample data from the following three countries: Korea, China, and Japan. Two evenly distributed samples were taken from each of the color variants of Korea. Only three green color individuals were gathered from China. From Japan, the numbers of samples for the red, green, and black variants are one, three, and one, respectively. We observed that the geographically segregated individuals were not clustered together (Figure 1), indicating that geographic distance is not coupled with genetic differentiation. Taken together, our results suggest that the color variants of the A. japonicus populations are not monophyletic.

In conclusion, to explore and assign a taxonomic status to the three color variants of $A$. japonicus, we generated the phylogenetic trees of the family Stichopodidae based on COI and $16 S$ rRNA sequences using multiple phylogenetic analysis approaches. After considering the caveats and limitations that are associated with the use of the incorrect phylogenetic analysis and the selection of inappropriate outgroups, our results strongly support the single-species hypothesis for the three color variants of A. japonicus, and also confirm their non-monophyletic origin. As a result, the conclusions presented in this study will help to establish the rapidly growing sea cucumber aquaculture industry and the corresponding breeding programs.

\section{Reference}

1. Choe S, Oshima $Y$ (1961) On the morphological and ecological differences between two commercial forms, "Green" and "Red", of the Japanese common 
Citation: Jo J, Park C, Kim M, Park C (2016) Phylogenetic Analysis of the Three Color Variations of the Sea Cucumber Apostichopus japonicus. J Aquac Res Development. 7: 418. doi:10.4172/2155-9546.1000418

Page 4 of 4

sea cucumber, Stichopus japonicus Selenka. Nippon Suisan Gakkaishi 27: $97-$ 106.

2. Kanno M, Kijima A (2002) Quantitative and qualitative evaluation on the color variation of the Japanese sea cucumber Stichopus japonicus. Suisanzoshoku 50: 63-69.

3. Hongsheng Y, Annie M (2015) The Sea Cucumber Apostichopus japonicus: History, Biology and Aquaculture. Academic Press 37-52.

4. Bordbar S, Anwar F, Saari N (2011) High-value components and bioactives from sea cucumbers for functional foods: a review. Mar Drugs 9: 1761-1805.

5. Purcell S (2014) Value, market preferences and trade of Beche-de-mer from Pacific Island sea cucumbers PLoS One 9: e95075.

6. Sun X, Li Q, Kong L (2010) Comparative mitochondrial genomics within sea cucumber (Apostichopus japonicus): Provide new insights into relationships among color variants. Aquaculture $309: 280-285$.

7. Zhang W, Cao Z, Li Y, Zhao H, Haung J, et al. (2015) Taxonomic status of the three color variants in sea cucumber (Apostichopus japonicus): evidence from mitochondrial phylogenomic analyses. Mitochondrial DNA 1-4.

8. Nei M, Gojobori T (1986) Simple methods for estimating the numbers of synonymous and nonsynonymous nucleotide substitutions. Mol Biol Evol 3: 418-426.

9. Kimura M (1980) A simple method for estimating evolutionary rates of base substitutions through comparative studies of nucleotide sequences. J Mol Evol 16: $111-120$.

10. Ware J, Jesse L, Klaus K, Lauren S (2008) Relationships among the major lineages of Dictyoptera: the effect of outgroup selection on dictyopteran tree topology. Systematic Entomology 33: 429-450
11. Lyons-Weiler J, Hoelzer G, Tausch R (1998) Optimal outgroup analysis. Biological Journal of the Linnean Society 64: 493-511.

12. Dalevi D, Hugenholtz P, Blackall L (2001) A multiple-outgroup approach to resolving division-level phylogenetic relationships using $16 \mathrm{~S}$ rDNA data. Int $\mathrm{J}$ Syst Evol Microbiol 51: 385-391.

13. Sanderson M, Shaffer H (2002) Troubleshooting molecular phylogenetic analyses. Annual Review of Ecology and Systematics 33: 49-72.

14. Arndt A, Carios, Philip L, Michael J (1996) Molecular phylogeny of eastern Pacific sea cucumbers (Echinodermata: Holothuroidea) based on mitochondria DNA sequence. Mol Phylogenet Evol, 1996. 6: 425-437.

15. Kerr A, Daniel A, Ronald M, Yves S, Jeni K, et al. (2005) Molecular phylogeny of coral-reef sea cucumbers (Holothuriidae: Aspidochirotida) based on $16 \mathrm{~S}$ mitochondrial ribosomal DNA sequence. Mar Biotechnol 7: 53-60.

16. Thompson J, Higgins D, Gibson T (1994) Clustal W: improving the sensitivity of progressive multiple sequence alignment through sequence weighting, position-specific gap penalties and weight matrix choice. Nucleic Acids Re0search 22: 4673-4680.

17. Tamura K (2013) MEGA6: Molecular Evolutionary Genetics Analysis version 6.0. Mol Biol Evol 30: 2725-2729.

18. Uthicke S, Byrne M, Conand C (2010) Genetic barcoding of commercial Bechede-mer species (Echinodermata: Holothuroidea). Mol Ecol Resour 10: 634646.

19. Yang L, Tan Z, Wang D, Ling X, Guan M, et al. (2014) Species identification through mitochondrial rRNA genetic analysis. Sci Rep 4: 4089.

20. Soergel D (2012) Selection of primers for optimal taxonomic classification of environmental 16S rRNA gene sequences. ISME J 6: 1440-1444. 